\title{
Anxiety and Depression of the Patients with Hematological Malignancies during Hospitalization for Hematopoietic Stem Cell Transplantation
}

\author{
Ho-Jun Seo', Young-Gun Baek², Byung-Sik Cho ${ }^{3}$, Tae-Suk Kim4, Yoo Hyun Um¹, and Jeong-Ho Chae ${ }^{4 凶}$ \\ 'Department of Psychiatry, St. Vincent's Hospital, College of Medicine, The Catholic University of Korea, Suwon, Republic of Korea \\ 2Seoul Metropolitan Office of Education, Seoul, Republic of Korea \\ ${ }^{3}$ Department of Hematology, Catholic Blood and Marrow Transplantation Center, Seoul St. Mary's Hospital, Leukemia Research Institute, \\ College of Medicine, The Catholic University of Korea, Seoul, Republic of Korea \\ ${ }^{4}$ Department of Psychiatry, Seoul St. Mary's Hospital, College of Medicine, The Catholic University of Korea, Seoul, Republic of Korea
}

Objective This study aimed to assess the anxiety and depression in patients undergoing hematopoietic stem cell transplantation (HSCT). Methods Eighty-seven adult patients with various hematologic diseases, who were scheduled to receive autologous or allogeneic HSCT, were enrolled. The M.D. Anderson Symptom Inventory and the Hospital Anxiety Depression Scale were applied prospectively at hospital admission (D-14), on the day of transplantation (D day), and at 7 (D7) and 14 days (D14) after transplantation.

Results The severity of both anxiety and depressive symptoms increased over time, with a peak at D7, and then showed a downturn at D14. Physical distresses also started with mild intensity at base line, which were continuously aggravated until D7, and then a partial recovery afterwards. Approximately, 52\% of the participants had significantly high anxiety or depression before the start of HSCT. The occurrence of aggravation of pain, nausea, shortness of breath, and lack of appetite was associated with the development of anxiety during isolation period. The patients with significant baseline anxiety had higher scores on fatigue and shortness of breath items at D7 compared to those without.

Conclusion Our finding suggests the importance of psychiatric approaches, including preventive measures, for the patients undergoing HSCT.

Psychiatry Investig 2019;16(10):751-758

Key Words Hematopoietic stem cell transplantation, Anxiety, Depression, Physical symptom burdens.

\section{INTRODUCTION}

Hematopoietic stem cell transplantation (HSCT) is a highly aggressive medical therapy among cancer treatments. Patients undergoing HSCT experience critical toxic side effects related to a rigorous conditioning regimen and graft-versushost disease (GVHD), with the risk of mortality from the procedure itself. ${ }^{1}$ HSCT requires a long hospital stay with isolation of at least 4 weeks and frequent extended recovery periods. Most patients already have experiences of several

\section{Received: June 2, 2019 Revised: July 1, 2019}

Accepted: July 12, 2019

$\triangle$ Correspondence: Jeong-Ho Chae, MD

Department of Psychiatry, College of Medicine, The Catholic University of Korea, 222 Banpo-daero, Seocho-gu, Seoul 06591, Republic of Korea

Tel: +82-2-2258-6083, Fax: +82-2-594-3870, E-mail: alberto@catholic.ac.kr

(c) This is an Open Access article distributed under the terms of the Creative Commons Attribution Non-Commercial License (https://creativecommons.org/licenses/by$\mathrm{nc} / 4.0$ ) which permits unrestricted non-commercial use, distribution, and reproduction in any medium, provided the original work is properly cited. treatment failures and a relapse of cancer and seem to suffer from physical and psychological distress even before the start of HSCT. ${ }^{2,3}$ Due to these specific issues for HSCT, several studies have directly focused on symptoms of anxiety and depression and the occurrence of psychological morbidity during HSCT, rather than assessing global quality of life as is generally used for patients with cancer. ${ }^{4-9}$

Previous researches have shown that approximately $20 \%$ of patients develop clinically significant psychiatric disorders, such as depressive disorder, anxiety disorder, or adjustment disorder with anxiety or depressed mood during hospitalization for HSCT. ${ }^{6,10}$ In addition to deleterious effects on quality of life, ${ }^{9,11}$ anxiety and depression seem to negatively affect subjective symptom burden, compliance with medical treatment, and length of hospital stay in patients undergoing HSCT. ${ }^{12,13}$ Major depression during hospitalization is also predictive of higher mortality 1 - and 3 year after HSCT. ${ }^{14}$ A recent research reported that many patients experienced the hospitalization as 
a traumatic event and approximately $30 \%$ of HSCT patients meet the criteria for PTSD at 6 months after HSCT. ${ }^{15}$

Although numerous studies have reported the quality of life and physical or psychological burden of the survivors of HSCT, prospective data which examine the experience of patients during hospitalization for HSCT remain scarce. ${ }^{4,5,8,9}$ Although there is a general acceptance that an aggravation of physical condition is expected to affect psychological burden during HSCT, the association has not been well recognized. Previously, Anderson et al. ${ }^{16}$ prospectively assessed the severity of individual symptoms during hospitalization in detail with specific measures designed for HSCT patients. This research found that most physical symptoms were most intense at 3-10 days after transplantation. Contrary to consistent findings of physical burden, prospective studies have shown inconsistent results on time course of anxiety and depression during hospitalization. ${ }^{4,5,8,9}$ Especially, depression and anxiety each has shown slightly different patterns according to studies. ${ }^{5,89}$ Moreover, besides physical and psychological burden related to HSCT and isolation, several researches have shown that substantial portion of patients already experience intense distress even before the start of HSCT. Prieto et al. ${ }^{13}$ found that 46 of 220 (21\%) patients met the criteria for depressive, anxiety or adjustment disorder at the day of hospital admission. Two cross sectional studies found that more than half of patients exceeded the threshold for clinically significant level of anxiety and/or depression at the pre-transplant assessments. ${ }^{2,3}$ A previous study found that anxiety and depression before hospitalization were predictors of high anxiety and depression during isolation periods. ${ }^{8}$ However, data are still insufficient to examine the relationship between patient's baseline psychological distress and their physical and psychological burdens during hospitalization.

We conducted a prospective longitudinal study to assess psychological and physical symptom burdens experienced by patients during hospitalization for HSCT. In this study, we also aimed to identify the association between aggravation of anxiety and depressive symptoms and that of physical symptom burdens. We also sought to investigate the impact of anxiety and depression before the start of HSCT on the clinical course of psychological and physical distress during hospitalization.

\section{METHODS}

\section{Participants}

The subjects were 87 patients with hematologic disease who were scheduled to receive autologous or allogeneic HSCT at St. Mary's Hospital, The Catholic University of Korea in Seoul, Korea. The patients were recruited from the outpatient clinic after they decided to undergo HSCT treatment following a clinician's recommendation. Inclusion criteria were transplant eligible patients with hematologic diseases, at least 18 years of age, and the ability to understand the research and to give consent to participate in the study. Of the 116 eligible patients, 93 agreed to participate and provided informed consent. Six could not be assessed due to a change in treatment schedule or a withdrawal from participation. Finally, 87 patients participated in the first assessment.

All patient transplants were performed in laminar airflow, high-efficiency particulate air-filtered rooms until engraftment. In cases of allogeneic HSCT, GVHD prophylaxis was attempted with cyclosporine (for sibling donors) or tacrolimus (for unrelated donors) and short-course methotrexate. Granulocyte-colony-stimulating factor was administered subcutaneously to all patients from day 7 after the transplant until neutrophil recovery. Low-dose heparin or lipo-prostaglandin E1 was administered with ursodiol to prevent venoocclusive disease. Antimicrobial prophylaxis consisted of ciprofloxacin and intraconazole started at the beginning of the conditioning treatment. Cytomegalovirus prophylaxis consisted of high-dose IV acyclovir until engraftment for all patients. Every patient received Pneumocystis jirovecci prophylaxis with sulfamethoxazole/trimethoprim after engraftment until discontinuation of the immunosuppressant.

Among the 87 participants, 14 dropped out by day 14 (six at D day, seven at D7, and one at D14). Participants were lost due to compromised medical status ( $n=6$, including one death) or a withdrawal from participation $(n=8)$.

This study was approved by the Institutional Review Board of St. Mary's Hospital, The Catholic University of Korea. All participants signed an informed consent form after receiving a full explanation of the procedure. All research was undertaken in accordance with the latest version of the Declaration of Helsinki (SCMC070T047).

\section{Study procedures}

Demographic and baseline clinical characteristics were assessed at pre-visit interviews before admission for HSCT. The study instruments were applied at hospital admission (D-14, day-10 to day-14 prior to transplantation, depending on the conditioning regimen), and subsequently on a weekly basis from the day of transplantation (D day) until discharge. Assessments were performed within 2 days of the scheduled interview. Similar to previous studies, only the data of D-14, D day, D7, and D14 were used in the analysis due to high dropout rates from discharge. ${ }^{4,5}$

The M.D. Anderson Symptom Inventory (MDASI) ${ }^{17}$ and Hospital Anxiety Depression Scale (HADS $)^{18}$ were used to assess multiple symptoms and emotional problems during HSCT. 
The MDASI was developed to measure the symptom burden during daily life in patients with cancer, including symptom severity and symptom-related interference with daily life. The MDASI assesses a patient's health status within the 24 hours prior to evaluation and includes individual symptom items for measuring symptom intensity and items that assess symptomrelated interference. Each item is rated on a scale of 0 (not at all) to 10 (as bad as you can imagine or completely interferes). The validity and reliability of the MDASI and the Korean version of the MDASI have been established. ${ }^{17,19}$ For the current study, 6 physical symptom items which are common in patients undergoing HSCT were used in the analysis. ${ }^{16,20}$ It includes pain, fatigue, nausea, shortness of breath, lack of appetite, and disturbed sleep. The HADS is a self-rating scale designed to screen for psychiatric morbidity in patients with medical illness. The HADS does not include the numerous somatic items found in the usual psychiatric measures that assess anxiety and depression. The HADS consists of two separate scales for anxiety and depressive symptoms, with seven items for each scale. Each item has a four-point Likert scale from 0 to 3, and the total score for anxiety or for depressive symptoms ranges from 0 to 21. HADS has been used frequently in patients with cancer, and the validity and reliability of the original version and the Korean version of HADS have been established. ${ }^{18,21,22}$ For the current study, the HADS assessed a patient's status within the 24 hours prior to evaluation and used a cutoff score of 7 .

\section{Data analysis}

Descriptive statistics were calculated for demographic and clinical characteristics of the participants. A mixed model for repeated measurement (MMRM) analysis was used to evaluate changes in HADS and MDASI symptom intensities and to evaluate group differences between those who had significant anxiety or depression prior to HSCT and those not, with diagnoses, HSCT methods, gender, and age as covariates. Pairwise logistic regression models were used to assess the association of MDASI symptom items with the occurrence of new anxiety and depression during HSCT. The individual MDASI symptoms item score at D7 was compared between the patients with and without significant anxiety/depression at D-14 using an analysis of covariance (ANCOVA) with the baseline scores, diagnoses, HSCT methods, gender, and age as covariates. All statistical tests were performed using SAS version 8.0 (SAS, Inc., Cary, NC, USA), with a two-tailed value of 0.05 , and the power of the sample to detect an effect size was $80 \%$.

\section{RESULTS}

\section{Participants' characteristics}

The sociodemographic and disease-related characteristics of the subjects are given in Table 1 . The mean age of the subjects was 38.1 years $(\mathrm{SD}=14.1)$, and the proportion of females was $42.5 \%$. The median time since diagnosis was 6 months

Table 1. Sociodemographic and disease-related characteristics of the participants $(\mathrm{N}=87)$

\begin{tabular}{|c|c|c|}
\hline Characteristic & $\mathrm{N}$ & $\%$ \\
\hline \multicolumn{3}{|l|}{ Age, years } \\
\hline \multicolumn{3}{|l|}{ Mean (SD): 38.1(14.1) } \\
\hline \multicolumn{3}{|l|}{ Range: $18-65$ years } \\
\hline \multicolumn{3}{|l|}{ Gender } \\
\hline Male & 50 & 57.5 \\
\hline Female & 37 & 42.5 \\
\hline \multicolumn{3}{|l|}{ Marital status } \\
\hline Married & 54 & 62.1 \\
\hline Unmarried & 30 & 34.5 \\
\hline Divorced or widowed & 3 & 3.4 \\
\hline \multicolumn{3}{|l|}{ Religion } \\
\hline None & 39 & 44.8 \\
\hline Protestant & 17 & 19.5 \\
\hline Buddhism & 12 & 13.8 \\
\hline Catholic & 18 & 20.7 \\
\hline Others & 1 & 1.1 \\
\hline \multicolumn{3}{|l|}{ Education } \\
\hline Less than high school & 13 & 14.9 \\
\hline High school degree & 32 & 36.8 \\
\hline Above college & 42 & 48.3 \\
\hline \multicolumn{3}{|c|}{ Economic status (below $2 / 3$ of median wage) } \\
\hline Previous psychiatric treatment & 21 & 24.1 \\
\hline Time since diagnosis, months & 3 & 3.4 \\
\hline \multicolumn{3}{|l|}{ Median: 6} \\
\hline \multicolumn{3}{|l|}{ IQR: 5-15 } \\
\hline \multicolumn{3}{|l|}{ Diagnosis } \\
\hline Acute myelogenous leukemia & 22 & 25.3 \\
\hline Acute lymphoblastic leukemia & 26 & 29.9 \\
\hline Multiple myeloma & 16 & 18.4 \\
\hline Non-Hodgkin's lymphoma & 2 & 2.3 \\
\hline Severe aplastic anemia & 10 & 11.5 \\
\hline Myelodysplastic syndromes & 8 & 9.2 \\
\hline Chronic myelogenous leukemia & 3 & 3.4 \\
\hline \multicolumn{3}{|l|}{ Type of HSCT } \\
\hline Autologous & 31 & 35.6 \\
\hline Allogeneic & 56 & 64.4 \\
\hline \multicolumn{3}{|l|}{ Conditioning regimen } \\
\hline Chemotherapy only & 28 & 32.2 \\
\hline
\end{tabular}

IQR: interquartile range, HSCT: hematopoietic stem cell transplantation 
(interquartile range, 5 to 15). Of the 87 patients, 26 suffered from acute lymphoblastic leukemia, 22 from acute myelogenous leukemia, 16 from multiple myeloma, 10 from severe aplastic anemia, 8 from myelodysplastic syndromes, 3 from chronic myelogenous leukemia, and 2 from non-Hodgkin's lymphoma. Approximately $68 \%$ of the subjects were conditioned with chemoradiotherapy, and the other $32.2 \%$ with chemotherapy only. Approximately, 64\% of the subjects underwent allogeneic HSCT, and the remaining 35.6\% underwent autologous HSCT.

\section{Anxiety and depression during isolation period}

Figure 1 shows the mean scores of HADS and anxiety or depression cases with a cutoff score of 7 across the four time points. The severity of both anxiety and depressive symptoms increased over time, with a peak at D7, and then showed a downturn at D14. Depression showed a sharper increase over time compared with anxiety level. A significant main effect of time was observed for depression, but not for anxiety ( $p=0.003$ and $p=0.100$, respectively). The number of HADS anxiety and depression cases across four time points showed a similar trend to the time course of mean HADS scores, with a peak occurrence at D7. Before the start of HSCT, as many as $33.3 \%$ and $43.7 \%$ of the subjects were already included in the HADS anxiety and depression cases, respectively, and 51.7\% of the participants had significantly high anxiety or depression at baseline.

\section{The physical symptom burdens during isolation period}

Table 2 provides mean scores of 6 physical symptom items of MDASI across four time points. All symptom items showed a similar pattern of symptom change: mild symptom intensity at baseline, continuously aggravated symptoms until D7, and then a partial recovery. Significant main effects of time were observed for all of these symptoms.

\section{The associations between newly developed anxiety or depression and aggravation of physical symptoms}

Table 3 presents the results of pairwise logistic regression
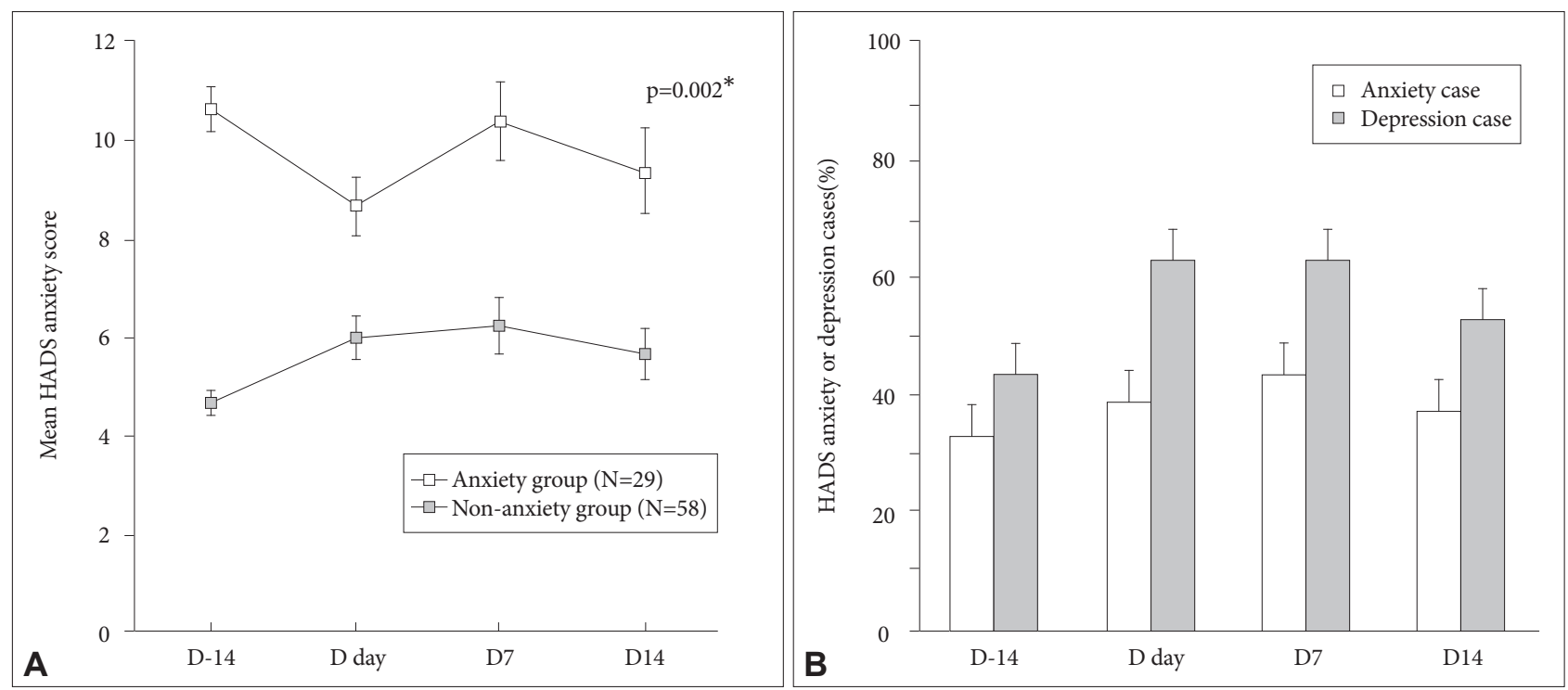

Figure 1. Mean scores and cases (\%) of HADS at different stage of the isolation periods*. A: HADS anxiety or depression cases were defined as HADS anxiety or depression scores $>7$, respectively. B: The main time effect in MMRM analysis. HADS: Hospital Anxiety Depression Scale.

Table 2. Mean (SD) scores of 6 physical symptom items of MDASI at different stage of the isolation periods

\begin{tabular}{lccccc}
\hline \multicolumn{1}{r}{ Items of MDSAI } & D-14 & D day & D7 & D14 & $\mathrm{p}^{\dagger}$ \\
\hline Pain & $2.58(3.20)$ & $4.22(2.85)$ & $5.95(3.14)$ & $4.93(3.24)$ & $<0.001^{*}$ \\
Fatigue & $3.63(2.89)$ & $4.55(2.88)$ & $5.39(2.83)$ & $4.70(2.65)$ & $<0.001^{*}$ \\
Nausea & $2.10(2.89)$ & $5.21(2.19)$ & $5.51(3.62)$ & $4.13(3.34)$ & $<0.001^{*}$ \\
Shortness of breath & $1.54(2.51)$ & $2.11(2.54)$ & $2.90(3.04)$ & $2.06(2.73)$ & $0.001^{*}$ \\
Lack of appetite & $3.13(3.25)$ & $4.71(3.20)$ & $5.07(3.36)$ & $4.70(3.03)$ & $<0.001^{*}$ \\
Disturbed sleep & $3.46(3.33)$ & $4.78(3.19)$ & $4.87(3.19)$ & $4.08(3.10)$ & $<0.001^{*}$ \\
\hline
\end{tabular}

${ }^{*} \mathrm{p}<0.01,{ }^{\dagger}$ the main time effect in MMRM analysis. MDASI: M.D. Anderson Symptom Inventory 
analysis of newly developed anxiety/depression and the aggravation of physical symptoms during isolation period. After the start of HSCT, anxiety and depression newly devel-

Table 3. The association between newly developed anxiety or depression and aggravation of physical symptoms during isolation periods*

\begin{tabular}{lcccccc}
\hline \multirow{2}{*}{ Items of MDSAI } & \multicolumn{2}{c}{ New anxiety } & & \multicolumn{2}{c}{ New depression } \\
\cline { 2 - 3 } \cline { 5 - 6 } \cline { 5 - 6 } Pain & OR & $95 \%$ CI & & OR & $95 \%$ CI \\
\hline Fatigue & 3.30 & $1.16-9.41$ & & - & - \\
Nausea & - & - & & - & - \\
Shortness of breath & 4.90 & $1.79-13.40$ & & - & - \\
Lack of appetite & 5.41 & $1.79-16.34$ & & - \\
Disturbed sleep & - & - & & - & - \\
\hline
\end{tabular}

*new anxiety/depression and aggravation of physical symptoms were defined as a case of HADS anxiety/depression scores $>7$ and scores of each symptom item in MDASI $>5$, respectively. MDASI: M.D. Anderson Symptom Inventory, HADS: hospital anxiety depression scale, OR: odds ratio, CI: confidence interval oped in $24(27.6 \%)$ and $32(36.8 \%)$ cases, respectively. The occurrence of significant aggravation of symptoms such as pain, nausea, shortness of breath, and lack of appetite was associated with the development of anxiety. In contrast, no physical symptom on MDASI was associated with the development of depression.

\section{Comparison between patients with significant anxiety or depression at baseline and those without}

Patients were divided into two groups, those who had significant anxiety or depression prior to HSCT (anxiety group or depression group) and those who did not (non-anxiety group or non-depression group), and their physical symptom scores on MDASI at D7, which was the most critical time point of distress, were compared (Table 4). When a ANCOVA was performed with baseline scores, diagnoses, HSCT methods, gender and age as covariates, the anxiety group had higher scores on individual symptom items of fatigue and shortness of breath compared to the non-anxiety group

Table 4. Comparison of mean scores (SD) of physical symptom burdens at D7 between the patients who had significant anxiety/depression prior to HSCT (anxiety/depression group) and not (no anxiety/no depression group $)^{\dagger}$

\begin{tabular}{lcccccc}
\hline Items of MDSAI & Anxiety group & Non-anxiety group & $\mathrm{p}$ & Depression group & Non-depression group & $\mathrm{p}$ \\
\hline Pain & $6.60(2.70)$ & $5.60(3.30)$ & 0.164 & $6.16(3.12)$ & $5.68(3.18)$ & 0.452 \\
Fatigue & $6.55(2.25)$ & $4.81(2.94)$ & $0.009^{* *}$ & $5.04(2.83)$ & $5.84(2.81)$ & 0.241 \\
Nausea & $6.28(3.26)$ & $5.12(3.75)$ & 0.186 & $5.67(3.48)$ & $5.29(3.83)$ & 0.690 \\
Shortness of breath & $3.93(3.31)$ & $2.38(2.78)$ & $0.020^{*}$ & $2.53(2.83)$ & $3.37(3.27)$ & 0.198 \\
Lack of appetite & $5.52(3.31)$ & $4.84(3.39)$ & 0.365 & $4.90(3.29)$ & $5.29(3.49)$ & 0.589 \\
Disturbed sleep & $5.03(3.64)$ & $4.79(2.97)$ & 0.405 & $4.96(2.81)$ & $4.76(3.66)$ & 0.411 \\
\hline
\end{tabular}

${ }^{*} \mathrm{p}<0.05,{ }^{* *} \mathrm{p}<0.01,{ }^{\dagger}$ ANCOVA was performed with baseline scores, diagnoses, HSCT methods, sex and age as covariates. HSCT: hematopoietic stem cell transplantation

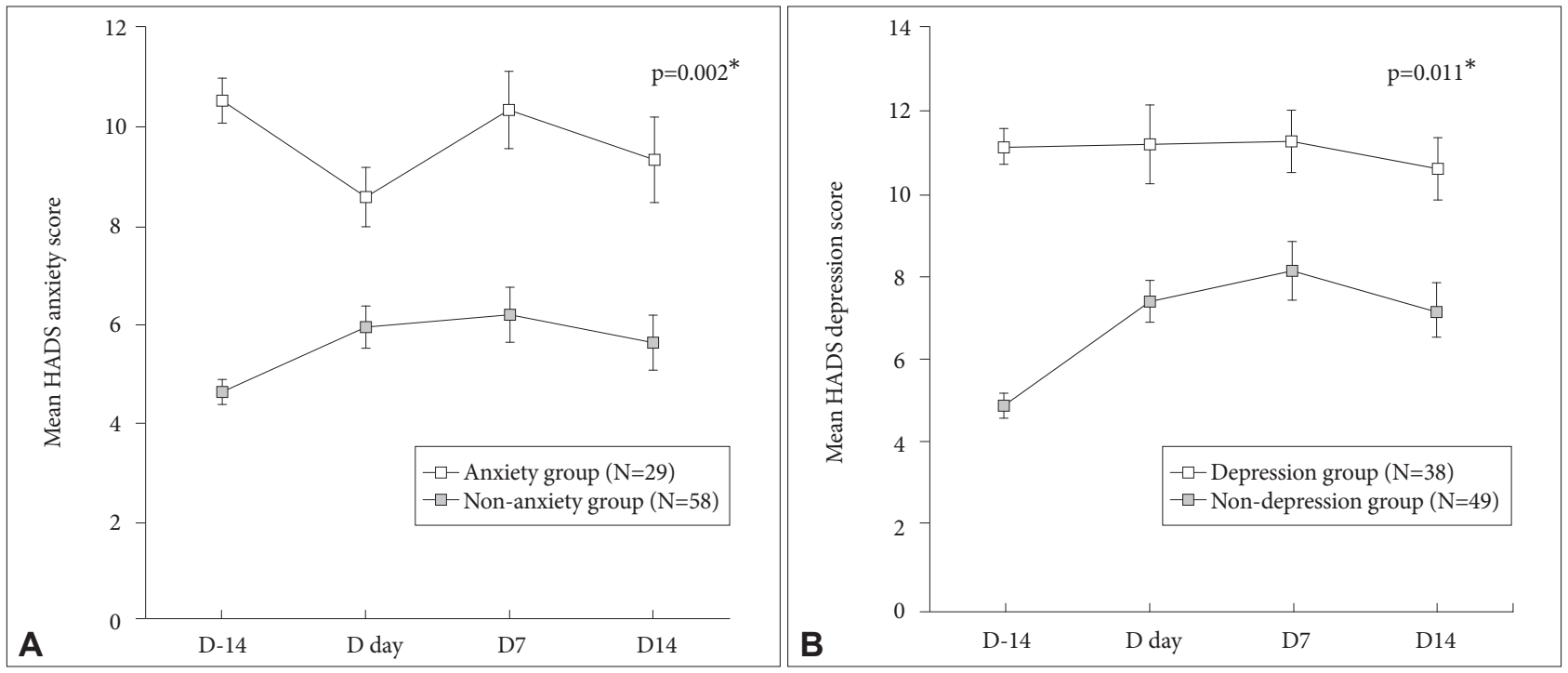

Figure 2. HADS anxiety and depression scores across 4 time points by anxiety and non-anxiety group and by depression and non-depression group. A: HADS anxiety scores of anxiety and non-anxiety group. B: HADS depression scores of depression and non-depression group. *time by group effects in MMRM analysis using diagnoses, HSCT method, gender, and age as covariates. HADS: hospital anxiety depression scale, HSCT: hematopoietic stem cell transplantation. 
( $\mathrm{p}=0.009$ and $\mathrm{p}=0.020$, respectively). However, there was no significant difference between the depression group and the non-depression group.

Figure $2 \mathrm{~A}$ illustrates the varying patterns of anxiety during isolation period in the anxiety and the non-anxiety groups. The anxiety level of those in the anxiety group was highest at baseline, decreased at D-day, was aggravated at D7, and slightly recovered at D14. However, only minimal changes in anxiety over time were observed in the non-anxiety group. The course of anxiety revealed a significant difference between the anxiety and non-anxiety groups using diagnoses, HSCT method, gender, and age as covariates (time by group interaction, $\mathrm{p}=0.002$ ). Depression severity also revealed a significant difference between the depression and non-depression groups (time by group interaction, $\mathrm{p}=0.011$ ) (Figure $2 \mathrm{~B}$ ) The depression group reported a high level of depression continuously throughout the study period, with minimal change over time. In contrast, the non-depression group reported mild depression at baseline, but showed a sharp increase in severity of depression until $\mathrm{D} 7$, which partially recovered at D14. Despite the fact that their global depression level was lower than that in the depression group, the non-depression group experienced quite a severe aggravation of depression over time compared with the depression group.

\section{DISCUSSION}

In the current study, the courses of physical symptom burden of the patients undergoing HSCT showed a gradual increase in symptom intensity from baseline to a peak at 7 days after transplantation. It is consistent with the majority of prospective studies which used similar assessment time points. ${ }^{5,916}$ We found the severity of anxiety and depression symptoms were also continuously aggravated until 7 days after transplantation, and both were partially recovered by 14 days after transplantation. The previous prospective findings of anxiety and depression have somewhat varied across studies. Fife et al. ${ }^{4}(n=97)$ found that both anxiety and depressive symptoms increased from baseline, with the highest intensity at 1 day before transplantation, and then decreased gradually until 14 days after transplantation. In contrast, Prieto et al. ${ }^{5}$ $(n=220)$ found discrepancies between the time courses of anxiety and depressive symptoms. The score for anxiety was highest at hospital admission and decreased gradually throughout HSCT, whereas depression showed a sharp increase from baseline until 7 days after transplantation and decreased after that. Tecchio et al. ${ }^{8}(\mathrm{n}=107)$ and El-Jawahri et al. ${ }^{9}(\mathrm{n}=90)$ found a sharp aggravation of depression from baseline until 1 week after transplantation, while the severity of anxiety was stable from baseline throughout the study periods. It is inter- esting to note that despite varied findings of the temporal trajectory of anxiety from a stable course to a reduction over the HSCT periods, depression level is relatively concordant, which is usually severe during the isolation periods. Consistent with previous findings, depression level in the current study showed a steeper increase compared to anxiety. The MMRM showed significant main effect of time for depression, but not for anxiety. The rates of patients with HADS depression scores $>7$ climbed from $42.7 \%$ up to $63.2 \%$ until 7 days after transplantation, whereas the rates of those with HADS anxiety scores $>7$ changed only from $33.3 \%$ to $39.1 \%$.

In the current study, substantial number of patients already had significant level of anxiety and depression even before the start of transplantation. We found approximately half of the participants had HADS anxiety or depression scores $>7$ at baseline. This finding is notable given that only 3 participants had the past psychiatric history. Our finding seems rather higher compared to previous prospective findings. $4,5,8,9$ The reason is unclear. However, compared with previous studies, the participants of the current study had relatively a short median time since diagnosis (6 months), which might be not enough to accept and cope with their disease. Whereas, consistent with our finding, previous studies of pre-transplantation evaluation found that more than half of the patients had clinically significant anxiety or depression level. ${ }^{2,3}$ High pre-transplant anxiety or depression is a predictive factor for emotional distress during HSCT, and anxiety or depression before or during HSCT is also predictive of earlier mortality of HSCT. ${ }^{11,14,23}$ Our findings suggest that the cumulative psychological burden from the course of chemotherapy for induction and consolidation could lead some vulnerable patients to a significant level of distress even before the start of HSCT.

It is interesting to note the difference of time course of emotional distress between patients with baseline anxiety or depression and those without. Figure 2 shows that patients with baseline anxiety experienced their most severe anxiety at the moment of admission, which might reflect their uncertainty and fearfulness confronted with the highly aggressive medical therapy. ${ }^{5}$ Since then, the anxiety level was decreased at the day of transplantation, then aggravated again at 7 days after transplantation, and then slightly recovered at 14 days after transplantation, which might follow the pattern of physical burden. Whereas, only a slight change in anxiety was observed in patients without baseline anxiety. It suggests patients without severe anxiety at the beginning of transplant procedure might handle their anxiety well during isolation period. With regards to depression, patients with baseline depression reported a high level of depression continuously with minimal change throughout HSCT. On the other hand, the de- 
pression level in patients without baseline depression was mild at the moment of admission, but showed rather a sharp increase until 7 days after transplantation, and then partially decreased. This finding suggests that even patients who do not have significant depression at the start of HSCT might experience quite severe aggravation of depression during HSCT. Interestingly, previous studies have found that rather than biomedical variables, non-biological variables such as personality trait, pre-transplant anxiety or depression, and past psychiatric history to be the most independent predictors for emotional distress during HSCT., ${ }^{8,10,12,13}$ Focusing on the patients with high anxiety or depression at the very beginning of transplant procedure might be a useful method to screen a possible case at risk of developing severe anxiety or depression during HSCT. Our finding also suggests that in contrary to anxiety, attention is needed even in the patients without high baseline depression. Empirical data from future studies are needed for the confirmation of this finding.

Patients with baseline anxiety experienced significantly higher distress in the MADSI items of fatigue and shortness of breath at D-7, the most critical time point for distress from HSCT. No significant difference was observed between patients with baseline depression and those without. Additionally, aggravation of various symptoms, such as pain, nausea, shortness of breath, and lack of appetite were associated with newly emerged anxiety during isolation periods, whereas no MADSI symptom items were associated with newly-emerging depression. Numerous studies in cancer patients found that physical symptom intensity is associated with psychological factors such as anxiety and depression. ${ }^{24-27}$ Modulations of pain signal, expectation of pain, ability to cope/self-efficacy, personality traits and sleep have been known as possible mediating factors between physical symptom and anxiety/depression. ${ }^{28}$ However the relationships are complex and difficult to fully characterize. To our knowledge, few studies have examined the distinct impact of anxiety and depression on the perception of physical stimuli. A previous study in 405 patients with cancer, which included three subgroups of depression and anxiety, depression but not anxiety, and neither, revealed that anxiety and depression had additive and independent effects on somatic symptom burden. ${ }^{29}$ Nevertheless, the reason of our finding that the distress from various physical symptoms had a stronger association with anxiety than with depression is unclear. A recent literature highlighted the importance of the meaning of events to an individual as an important factor in making cancer patients anxious. ${ }^{30}$ Supposing that patients undergoing HSCT might already know well and worry about their treatment related mortality and life-threatening complications, those with higher anxiety might be more sensitive to their physical reactions during HSCT and feel more distressed. Moreover, emerging side effects may be interpreted more catastrophically, thereby worsening their anxiety rather than depression as an acute response. Further data is needed to replicate this finding in the future.

The current study had several limitations. Generalization of the results should be restricted because the sample size was small, and the study was performed at a single institution. The medication such as benzodiazepines and hypnotics were prescribed on an as-needed basis for patients, which could have affected the response to the assessments of psychological and physical distress. In the current study, to minimize patient burden during HSCT, only two subjective measures were used to assess distress during HSCT. Therefore, only small amounts of data were provided compared with previous prospective studies. However, considering the great degree of side effects such as fever, nausea, and GVHD, even a small amount of paperwork may have been difficult for the patients. Even in healthy populations, the number of questions in scales negatively influences the accuracy of answers. ${ }^{31}$ The simplicity of the assessments in the current study may have had positive effects on the reliability of the data compared with the previous prospective studies. ${ }^{4,5,8,9}$

Despite these limitations, the current study has significant meanings. It contributes to the prospective data of patients who are most difficult to engage in studies due to the considerable burden of side effects. The current results highlight the importance of the psychological distress in patients with hematologic disease and provide a basis for further studies on the benefits of the psychiatric treatment prior to the start of HSCT. Studies examining the efficacy and safety of psychiatric approaches such as medication and psychotherapy before the start of HSCT and studies to distinguish patients who experience persistent psychological symptoms after HSCT are needed.

\section{Conflicts of Interest}

The authors have no potential conflicts of interest to disclose.

\section{Author Contributions}

Conceptualization: Ho-Jun Seo, Tae-Suk Kim, Jeong-Ho Chae. Data curation: Ho-Jun Seo, Young-Gun Baek. Formal analysis: Ho-Jun Seo, Jeong-Ho Chae. Investigation: Ho-Jun Seo, Young-Gun Baek. Methodology: Ho-Jun Seo, Byung-Sik Cho, Jeong-Ho Chae. Project administration: Ho-Jun Seo, Jeong-Ho Chae. Resources: Ho-Jun Seo, Byung-Sik Cho. Supervision: Byung-Sik Cho, Jeong-Ho Chae. Validation: Ho-Jun Seo, Yoo Hyun Um. Visualization: Ho-Jun Seo. Writing_original draft: Ho-Jun Seo. Writing_review \& editing: Yoo Hyun Um, Jeong-Ho Chae.

\section{ORCID iDs}

Jeong-Ho Chae https://orcid.org/0000-0002-6070-9324

Ho-Jun Seo https://orcid.org/0000-0003-1661-1394

\section{REFERENCES}

1. Funke VA, Moreira MC, Vigorito AC. Acute and chronic Graft-versus- 
host disease after hematopoietic stem cell transplantation. Rev Assoc Med Bras (1992) 2016;62(Suppl 1):44-50.

2. Lee SJ, Loberiza FR, Antin JH, Kirkpatrick T, Prokop L, Alyea EP, et al. Routine screening for psychosocial distress following hematopoietic stem cell transplantation. Bone Marrow Transplant 2005;35:77-83.

3. Trask PC, Paterson A, Riba M, Brines B, Griffith K, Parker P, et al. Assessment of psychological distress in prospective bone marrow transplant patients. Bone Marrow Transplant 2002;29:917-925.

4. Fife BL, Huster GA, Cornetta KG, Kennedy VN, Akard LP, Broun ER. Longitudinal study of adaptation to the stress of bone marrow transplantation. J Clin Oncol 2000;18:1539-1549.

5. Prieto JM, Atala J, Blanch J, Carreras E, Rovira M, Cirera E, et al. Patient-rated emotional and physical functioning among hematologic cancer patients during hospitalization for stem-cell transplantation. Bone Marrow Transplant 2005;35:307-314.

6. Sasaki T, Akaho R, Sakamaki H, Akiyama H, Yoshino M, Hagiya K, et al. Mental disturbances during isolation in bone marrow transplant patients with leukemia. Bone Marrow Transplant 2000;25:315-318.

7. Yalvac HD, Kotan Z, Tekgunduz E, Caykoylu A, Altuntas F. Could psychiatric assessment before hematopoietic stem cell transplantation predict the need for psychiatric consultation during transplantation period? Transfus Apher Sci 2016;54:85-90.

8. Tecchio C, Bonetto C, Bertani M, Cristofalo D, Lasalvia A, Nichele I, et al. Predictors of anxiety and depression in hematopoietic stem cell transplant patients during protective isolation. Psychooncology 2013; 22:1790-1797.

9. El-Jawahri AR, Traeger LN, Kuzmuk K, Eusebio JR, Vandusen HB, Shin JA, et al. Quality of life and mood of patients and family caregivers during hospitalization for hematopoietic stem cell transplantation. Cancer 2015;121:951-959.

10. Prieto JM, Blanch J, Atala J, Carreras E, Rovira M, Cirera E, et al. Stem cell transplantation: risk factors for psychiatric morbidity. Eur J Cancer 2006;42:514-520.

11. Loberiza FR Jr, Rizzo JD, Bredeson CN, Antin JH, Horowitz MM, Weeks JC, et al. Association of depressive syndrome and early deaths among patients after stem-cell transplantation for malignant diseases. J Clin Oncol 2002;20:2118-2126.

12. Schulz-Kindermann F, Hennings U, Ramm G, Zander AR, Hasenbring $\mathrm{M}$. The role of biomedical and psychosocial factors for the prediction of pain and distress in patients undergoing high-dose therapy and BMT/PBSCT. Bone Marrow Transplant 2002;29:341-351.

13. Prieto JM, Blanch J, Atala J, Carreras E, Rovira M, Cirera E, et al. Psychiatric morbidity and impact on hospital length of stay among hematologic cancer patients receiving stem-cell transplantation. J Clin Oncol 2002;20:1907-1917.

14. Prieto JM, Atala J, Blanch J, Carreras E, Rovira M, Cirera E, et al. Role of depression as a predictor of mortality among cancer patients after stem-cell transplantation. J Clin Oncol 2005;23:6063-6071.
15. El-Jawahri AR, Vandusen HB, Traeger LN, Fishbein JN, Keenan T, Gallagher ER, et al. Quality of life and mood predict posttraumatic stress disorder after hematopoietic stem cell transplantation. Cancer 2016;122:806-812.

16. Anderson KO, Giralt SA, Mendoza TR, Brown JO, Neumann JL, Mobley GM, et al. Symptom burden in patients undergoing autologous stem-cell transplantation. Bone Marrow Transplant 2007;39:759-766.

17. Cleeland CS, Mendoza TR, Wang XS, Chou C, Harle MT, Morrissey $\mathrm{M}$, et al. Assessing symptom distress in cancer patients: the M.D. Anderson Symptom Inventory. Cancer 2000;89:1634-1646.

18. Zigmond AS, Snaith RP. The hospital anxiety and depression scale. Acta Psychiatr Scand 1983;67:361-370.

19. Yun YH, Mendoza TR, Kang IO, You CH, Roh JW, Lee CG, et al. Validation study of the Korean version of the M. D. Anderson Symptom Inventory. J Pain Symptom Manage 2006;31:345-352.

20. Campagnaro E, Saliba R, Giralt S, Roden L, Mendoza F, Aleman A, et al. Symptom burden after autologous stem cell transplantation for multiple myeloma. Cancer 2008;112:1617-1624.

21. Oh SM, Min KJ, Park DB. A study on the standardization of the hospital anxiety and depression scale for Koreans. J Korean Neuropsychiatr Assoc 1999;38:289-295.

22. Herrmann C. International experiences with the Hospital Anxiety and Depression Scale--a review of validation data and clinical results. J Psychosom Res 1997;42:17-41.

23. Akaho R, Sasaki T, Mori S, Akiyama H, Yoshino M, Hagiya K, et al. Psychological factors and survival after bone marrow transplantation in patients with leukemia. Psychiatry Clin Neurosci 2003;57:91-96.

24. Poulos AR, Gertz MA, Pankratz VS, Post-White J. Pain, mood disturbance, and quality of life in patients with multiple myeloma. Oncol Nurs Forum 2001;28:1163-1171.

25. Sela RA, Bruera E, Conner-spady B, Cumming C, Walker C. Sensory and affective dimensions of advanced cancer pain. Psychooncology 2002;11:23-34.

26. Spiegel D, Sands S, Koopman C. Pain and depression in patients with cancer. Cancer 1994;74:2570-2578.

27. Zaza C, Baine N. Cancer pain and psychosocial factors: a critical review of the literature. J Pain Symptom Manage 2002;24:526-542.

28. Nicolson SE, Caplan JP, Williams DE, Stern TA. Comorbid pain, depression, and anxiety: multifaceted pathology allows for multifaceted treatment. Harv Rev Psychiatry 2009;17:407-420.

29. Brown LF, Kroenke K, Theobald DE, Wu J, Tu W. The association of depression and anxiety with health-related quality of life in cancer patients with depression and/or pain. Psychooncology 2010;19:734-741.

30. Stark DP, House A. Anxiety in cancer patients. Br J Cancer 2000;83: 1261-1267.

31. Lee KS, Song HJ, Sohn YW, Hwang MJ, Park YS. Cognitive approaches to survey methodology: How the number or order of choices affects answers. J Korean Offic Stat 2008;13:1-33. 